onset $\left(\mathrm{PR}_{\mathrm{adj}}\right.$ :1.23 among men; 1.54 among women), receiving condoms free of charge $\left(\mathrm{PR}_{\mathrm{adj}}: 1.39\right.$ among men; 1.37 among women); besides, among men: casual sexual partner in the previous year $\left(\mathrm{PR}_{\mathrm{adj}}: 1.24\right)$ and same-sex sexual partner $\left(\mathrm{PR}_{\mathrm{adj}}: 1.23\right)$; among women with the sexual onset after 15 yo $\left(\mathrm{PR}_{\mathrm{adj}}: 1.25\right)$. HIV-testing has displayed a negative association for women $\left(\mathrm{PR}_{\mathrm{adj}}\right.$ : 0.72).

Conclusion The condom is widely known, there is a pattern of it use during the first and last sexual intercourse; free condoms are important to improve it use by the youths, and people use condom as a risk management strategy. Condom as prevention strategy is still useful. After these results, the strategy of prevention was adopted as a public policy in the city of Sao Paulo: large condom dispensers were allocated in the 28 urban bus terminals, where 6 million people pass around daily and by the end of 2016, 80 million free condoms will be distributed.

\section{P3.61 TRENDS IN ADULT CHLAMYDIA AND GONORRHOEA PREVALENCE, INCIDENCE AND URETHRAL DISCHARGE CASE REPORTING IN MOROCCO OVER 1995-2015 - ESTIMATES USING THE SPECTRUM-STI MODEL}

${ }^{1}$ Eline Korenromp, ${ }^{2}$ Amina El-Kettani, ${ }^{3}$ Guy Mahiané, ${ }^{2}$ Aziza Bennani, ${ }^{4}$ Laith Abu-Raddad ${ }^{4}$ Alex Smolak, ${ }^{5}$ Jane Rowley, ${ }^{6}$ Nico Nagelkerke, ${ }^{7}$ Houssine El-Rhilani, ${ }^{7}$ Kamal Alami, ${ }^{8}$ Amina Hançali. ${ }^{1}$ Avenir Health, Geneva, Switzerland; ${ }^{2}$ Ministry of Health, Directorate of Epidemiology and Disease Control, Morocco, Rabat, Morocco; ${ }^{3}$ Avenir Health, Glastonbury (Ct), USA; ${ }^{4}$ Weill Cornell Medical College, Qatar, Cornell University, Doha, Qatar; ${ }^{5}$ Independent Consultant, London, UK; ${ }^{6}$ Malawi-Liverpool Wellcome Trust, Blantyre Malawi; ${ }^{7}$ UNAIDS Morocco Country Office, Rabat, Morocco; ${ }^{8}$ Stis Laboratory, Department of Bacteriology, National Institute of Hygiene, Rabat, Morocco

\subsection{6/sextrans-2017-053264.296}

Introduction Evolving health priorities and resource constraints mean that countries require data on trends in sexually transmitted infections (STI) burden, to inform program planning and resource allocation.

Methods The Spectrum modelling tool estimated prevalence and incidence of gonorrhoea and chlamydia in Morocco's 1549 year-old population, based on prevalence data adjusted for diagnostic test performance, urban/rural and male/female differences, and missing high-risk populations, and weighted by coverage and representativeness.

Results Gonorrhoea prevalence was estimated at 0.37\% (95\% confidence interval, $0.14 \%-1.0 \%$ ) in women and $0.32 \%$ $(0.12 \%-0.87 \%)$ in men in 2015 ; chlamydia prevalences were $3.8 \%(2.1 \%-6.4 \%)$ and $3.0 \% \quad(1.7 \%-5.1 \%)$. Corresponding numbers of new cases in 15-49 year-old women and men in 2015 were $79,598(23,918-256,206)$ and 112,013 (28,700$307,433)$ for gonorrhoea, and 291,908 (161,064-524,270) and $314,032(186,076-559,133)$ for chlamydia. Gonorrhoea and chlamydia prevalence had declined by $41 \%$ and $27 \%$, respectively, over 1995-2015. Prevalence declines probably related to improved STI treatment coverage, and decreasing risk behaviours. Reporting completeness among treated urethral discharge (UD) cases was estimated at 46\%-77\% in 2015. Clinically reported UD cases corresponded to $13 \%$ of all (symptomatic and asymptomatic) gonorrhoea and chlamydia cases.

Conclusion STI declines and improvements in treatment coverage are consistent with Morocco's introduction of syndromic management in 2000, scale-up of prevention interventions, and declining HIV incidence after 2003. While gonorrhoea is four-fold more common as cause of clinical UD cases than chlamydia, Morocco continues to suffer a large, untreated burden of chlamydia. Reliable monitoring of both STIs requires new periodic surveys and/or novel forms of affordable surveillance beyond high-risk populations.

\section{P3.62 ADULT PREVALENCE OF ACTIVE SYPHILIS IN LOW- AND MIDDLE-INCOME COUNTRIES, 1995-2016: BASELINE AND PROSPECT FOR REDUCTIONS TARGETED THROUGH THE GLOBAL STI CONTROL STRATEGY 2016-2021}

${ }^{1}$ Eline Korenromp, ${ }^{2}$ Guy Mahiané, ${ }^{3}$ Nico Nagelkerke, ${ }^{4}$ Jane Rowley, ${ }^{2}$ Carel Pretorius, ${ }^{5}$ Nicholas Kassebaum, ${ }^{6}$ Melanie Taylor, ${ }^{7}$ Laith Abu-Raddad. ${ }^{7}$ Avenir Health, Geneva, Switzerland; ${ }^{2}$ Avenir Health, Glastonbury, USA; ${ }^{3}$ Malawi-Liverpool Wellcome Trust, Blantyre - Malawi; ${ }^{4}$ Independent Consultant, Lonon, UK; ${ }^{5}$ Institute for Health Metrics and Evaluation, University of Washington and Seattle Children's Hospital, Seattle, USA; ${ }^{6}$ World Health Organisation, Geneva, Switzerland; ' Weill Cornell Medical College, Qatar, Cornell University, Doha, Qatar

\subsection{6/sextrans-2017-053264.297}

Introduction The World Health Organisation and many countries have set targets to reduce rates of syphilis and eliminate congenital syphilis. National trends in syphilis prevalence and incidence, however, are uncertain. We used the Spectrum-STI model to estimate prevalence trends of active syphilis in adult women, for countries with $\geq 3$ data points post-1999 including $\geq 1$ from year 2009 or later.

Methods Prevalence data were adjusted for diagnostic test performance, and for the contribution of high-risk populations under-represented in surveys. National trends were estimated by logistic regression, weighting each data point by its national coverage. Estimates for 117 countries were aggregated to regional totals, weighting each country by adult population size, and imputing for countries without a trend estimate using regional averages.

Results At January 2017, 1056 data points had been identified, between 1977-2016 (median 2009), covering 137 million tests (132 million in routine ANC screening, 5.3 million in ANC surveys; 0.79 million from non-ANC general adult population surveys), of which 1.36 million found active infection. In provisional estimates, prevalence was highest in sub-Saharan Africa and lowest in Europe and the Middle East and North Africa (MENA). Prevalence declined over 2002-2012 in all regions, with strongest proportional decline in MENA $(\geq 1 \%$ to $<0.1 \%)$ and largest absolute decline in Africa ( $\geq 3 \%$ to $1 \%-1.8 \%$ ). Trends over 2012-2016 remain to be confirmed with new data, including general population HIV and syphilis surveys conducted over 2015-2018 in some African countries.

Conclusion Spectrum-STI is a useful tool to interpret syphilis data in a systematic manner, and evaluate time trends in national adult syphilis prevalence; however some high-STI countries lack recent data from ANC or any other general populations. These first standardised country estimates suggests that syphilis declines need to accelerate in most countries, if by 2021 they are to meet impact targets of the global STI control strategy and eliminate congenital syphilis. 